\title{
PRODUÇÃO, FLUXO E ANÁLISE DE DADOS DO SISTEMA DE INFORMAÇÃO EM SAÚDE: UM CASO EXEMPLAR ${ }^{1}$
}

\author{
Geovana Hagata de Lima Souza Thaines², Roseney Bellato³, Ana Paula Silva de Faria ${ }^{4}$ Laura Filomena \\ Santos de Araújo ${ }^{5}$
}

\footnotetext{
${ }^{1}$ Estudo realizado como parte integrante do Sub-Projeto II da Pesquisa “Os desafios e perspectivas do SUS na atenção à saúde em municípios da área de abrangência da BR 163 no Estado de Mato Grosso" financiada pelo CNPq.

${ }^{2}$ Graduanda do Curso de Enfermagem da Faculdade de Enfermagem (FAEN) da Universidade Federal de Mato Grosso (UFMT). Bolsista PIBIC/CNPq. Mato Grosso, Brasil. E-mail: geohagata@gmail.com

${ }^{3}$ Doutora em Enfermagem. Docente da FAEN/UFMT. Mato Grosso, Brasil. E-mail: roseney@terra.com.br

${ }^{4}$ Mestre em Enfermagem. Enfermeira da Secretaria de Estado de Saúde de Mato Grosso. Mato Grosso, Brasil. E-mail: apaula. faria@gmail.com

${ }^{5}$ Doutora em Enfermagem. Docente da FAEN/UFMT. Mato Grosso, Brasil. E-mail: laurafil1@yahoo.com.br
}

RESUMO: Os Sistemas de Informação em Saúde, tem sido utilizados como ferramentas capazes de armazenar a produção de dados para subsidiar a avaliação em saúde. Indagamos como esses dados são produzidos e analisados e como o usuário é visualizado no sistema. Trata-se de um Estudo de Caso que possibilitou analisar a configuração da rede de informação em saúde a partir do desenho analítico sobre a produção, fluxo e análise dos dados nos diferentes níveis de atenção à saúde em relação ao Sistema de Informação da Atenção Básica e Sistema de Cadastro Nacional de Portadores de Hipertensão e Diabetes. Evidenciamos que os dados que compõem esses Sistemas de Informação em Saúde não estão sendo analisados, consequentemente, não permitem a análise situacional em saúde e/ou produção de informações para o planejamento da assistência e resolutividade das ações de saúde.

DESCRITORES: Sistema Único de Saúde. Serviços de saúde. Serviços de informação.

\section{PRODUCTION, FLOW AND ANALYSIS OF DATA INFORMATION SYSTEM FOR HEALTH: AN EXEMPLARY CASE}

\begin{abstract}
Information Systems in Health has been used as a tool capable of storing the production data and information to support assessment in health. We ask how these data are produced and interpreted and how the user is displayed in the System. This is a Case Study that has examined the configuration of the network of health information from the design analysis on the production and flow of data generated and analyzed in different levels of health care in the Information System of Primary Care System and National Register of Persons with Hypertension and Diabetes. Show that the data that comprise those in Health Information Systems do not allow the situational analysis on health and/or production of information on the planning of care and resolution of health actions.
\end{abstract}

DESCRIPTORS: Single Health System. Health services. Information services.

\section{PRODUCCIÓN, FLUJO Y ANÁLISIS DE DATOS DEL SISTEMA DE INFORMACIÓN PARA LA SALUD: UN CASO EJEMPLAR}

RESUMEN: Los Sistemas de Información en Salud han sido utilizados como una herramienta capaz de almacenar la producción de datos e información para apoyar la evaluación en salud. Para el presente estudio, investigamos cómo esos datos se producen y se interpretan, y cómo se muestra al usuario en el sistema. Es un Estudio de Caso en el que se ha examinado la configuración de la red de información para la salud, desde el diseño de análisis de la producción y el flujo de los datos generados y analizados en los diferentes niveles de atención a la salud en el Sistema de Información de Atención Primaria y del Registro Nacional de las Personas con Hipertensión y Diabetes. Los resultados muestran que los datos que componen esos Sistemas de Información en Salud no permiten el análisis de la situación en materia de salud y/o producción de información para la planificación de la atención y resolución de las acciones de salud.

DESCRIPTORES: Sistema Único de Salud. Servicios de salud. Servicios de información. 


\section{INTRODUÇÃO}

Comumente, dado e informação são considerados a mesma coisa. Porém, ao longo desta pesquisa, percebemos que há uma diferença importante entre esses conceitos e que se torna pertinente serem esclarecidos em suas peculiaridades, principalmente quando se refere a dados e informações em saúde, pois, são direcionadores de políticas de ação nesse setor.

O conceito de dado configura como uma sequência de símbolos quantificáveis que trazem na forma numérica a realidade em questão e que, por si só, não exprimem esta realidade; para que o dado faça sentido e expresse algo, é preciso que ele seja interpretado e analisado. ${ }^{1}$ Entretanto, o dado pode ser considerado como uma descrição limitada, enquanto que a informação se caracteriza como uma descrição mais ampliada baseada em um referencial explicativo. ${ }^{2}$ Sendo assim, os dados não são partes de uma informação, mas sim, de sua representação, que será interpretada e terá sentido de acordo com quem a interprete. ${ }^{1}$

No Sistema Único de Saúde (SUS), os dados são armazenados em bancos que permitem reunir vários dados previamente selecionados. OSistema de Informação em Saúde (SIS) reúne uma série de dados referentes a uma comunidade e a uma Política de Atenção à Saúde, por exemplo. Sendo assim, " $[. .$.$] os sistemas de informação de serviços$ de saúde são aqueles cujo propósito é selecionar os dados pertinentes a esses serviços e transformálos na informação necessária para o processo de decisões, próprio das organizações e indivíduos que planejam, financiam, administram, provêem, medem e avaliam os serviços de saúde" ${ }^{\prime 2: 26}$

A finalidade da informação em saúde consiste em identificar problemas individuais e coletivos do quadro sanitário de uma população, propiciando elementos para análise da situação encontrada e subsidiar o planejamento em saúde. ${ }^{2-3}$

Com a implantação do SUS, houve a necessidade de se criar SIS que fossem capazes de acompanhar toda a produção de dados e que assegurassem a realização de avaliações periódicas da situação de saúde no país. Deste modo, a estruturação dos SISs é de fundamental importância para o SUS monitorar as condições de saúde da população. ${ }^{4}$

O SUS possui inúmeros SISs, destacando-se os que abrangem atendimentos ambulatoriais e hospitalares, os de estatísticas vitais e de vigilância epidemiológica e sanitária. Dentre estes, existem os grandes SISs nacionais, como o Sistema de Informa- ção de Mortalidade (SIM), Sistema de Informação de Nascidos Vivos (SINASC), Sistema de Agravos de Notificação (SINAN), Sistema de Informação Ambulatorial (SIA), Sistema de Informação Hospitalar (SIH) e o Sistema de Informação da Atenção Básica (SIAB) e entre outros que são considerados elementos importantes para ações de controle, monitoramento e avaliação da atenção à saúde.

Em nosso estudo, focamos o nosso olhar na produção, fluxo e análise do banco de dados do SIAB e do Sistema de Cadastro Nacional de Portadores de Hipertensão e Diabetes (SIS/HIPERDIA). Na discussão, iremos descrever como compreendemos a lógica do SIS, tendo por base a pesquisa maior que este estudo se vincula realizada nos serviços de saúde no Município de Alta Floresta-MT.

O SIAB foi implantado em 1998 para substituir o Sistema de Informação do Programa de Agentes Comunitários de Saúde (SISPACS). O SIAB visa o acompanhamento das equipes do Programa Saúde da Família (PSF) e, por meio desse, obtêm-se dados sobre cadastros de famílias, condições de moradia e saneamento, situação de saúde, produção e composição das equipes de saúde, ou seja, oferece informações sobre a situação de saúde em que as famílias se encontram. ${ }^{5}$

O SIAB possui oito fichas de acompanhamento, entre elas, a ficha de hipertensos (B-HA), foco deste estudo; e a ficha de diabéticos (B-DIA), sendo que ambas tem como finalidade cadastrar e acompanhar mensalmente os usuários com diagnóstico confirmado, e a cada visita domiciliária realizada pelo Agente Comunitário de Saúde (ACS) os dados deverão ser atualizados. ${ }^{6}$

Na Atenção Básica, há outro SIS que também cadastra, acompanha e monitora os usuários com hipertensão arterial e Diabetes mellitus conforme as diretrizes do Plano de Reorganização da Atenção a Hipertensão Arterial e Diabetes mellitus. ${ }^{7}$ Este Plano foi implantado em janeiro de 2002 com objetivo de cadastrar os usuários com hipertensão arterial e Diabetes mellitus, e ofertar regularmente para a rede de Atenção Básica os medicamentos necessários a esses usuários, bem como, acompanhar e avaliar os índices de morbi-mortalidade destas doenças. No entanto, quando o usuário é diagnosticado como hipertenso e/ou diabético é preenchido a ficha de cadastramento do mesmo sendo que a primeira via fica anexada no prontuário e a segunda via é encaminhada para a Secretaria Municipal de Saúde (SMS) para a alimentação do banco de dados do SIS/HIPERDIA, sendo que este foi criado como 
uma ferramenta para o planejamento das ações em saúde aos usuários diagnosticados e cadastrados nesse SIS.7-8

O que nos chama a atenção é que tanto o SIAB e como SIS/HIPERDIA possuem banco de dados distintos que não se cruzam, sendo que esses dados são produzidos em um mesmo espaço e, ainda possuem dados em relação ao número de hipertensos, por exemplo, que diferem de um banco para outro.

Diante disso, indagamos como o usuário é visto pelo SIS e o modo como são produzidos e analisados os dados que alimentam os dois SISs para controle, monitoramento e planejamento da assistência aos usuários com hipertensão arterial e Diabetes mellitus na Atenção Básica.

Com base no exposto, este estudo teve como objetivo compreender a lógica existente na produção, fluxo e análise dos dados que alimentam o SIAB e o SIS/HIPERDIA, e a participação do nível municipal, estadual e federal na produção e análise dos dados, bem como o modo como retornam essas informações para os municípios. Outro ponto importante foi apreender como o usuário transita nestes SISs, ou seja, como ele é visto e representado em meio aos diversos números.

\section{METODOLOGIA}

Trata-se de um Estudo de Caso ${ }^{9}$ que permitiu a utilização de várias estratégias metodológicas e o aprofundamento necessário para compreender a produção, fluxo e análise dos dados que alimentam o SIAB e SIS/HIPERDIA desde a sua produção na Unidade Básica de Saúde (UBS) até alimentação do banco de dados no Ministério da Saúde (MS), dando especial destaque à forma como os dados entram no SIS e como o usuário é visualizado neles, tendo como caso exemplar o município e a microrregião de saúde de Alta Floresta-MT. O caso exemplar evidencia as regularidades das práticas que são demonstradas, não se desconsiderando, no entanto, as dissonâncias. Nele é feito uma seleção de fatos, ocorrências, ou acontecimentos que sintetizam significados importantes. ${ }^{10}$

A problematização deste estudo teve como ponto de partida a pesquisa na qual se abordou a trajetória assistencial de um usuário hipertenso residente em Alta Floresta-MT que esteve internado no município de Cuiabá-MT em busca por cuidados à sua saúde. ${ }^{16} \mathrm{~A}$ trajetória de busca pelo cuidado empreendida por este usuário foi refeita no presente estudo, com o intuito de apreender como se dão as práticas profissionais de atenção e de gestão em saúde nos locais por onde ele foi atendido até chegar a Cuiabá-MT, capital do nosso estado com ênfase na produção, fluxo e análise de dados.

Como estratégia metodológica, empregamos a observação participante, a seleção documental e a produção de imagem filmatográfica e fotográfica das práticas profissionais de atenção e de gestão em saúde realizadas por diferentes trabalhadores e nos diferentes serviços apontados na trajetória assistencial acima citada, que pudessem retratar o modo como a informação em saúde é produzida e utilizada nestas diferentes instâncias.

O trabalho de campo no município de Alta Floresta-MT foi realizado por um grupo de seis pesquisadores, no período de 16 a 20 de março de 2007. O local do estudo foi um Centro de Saúde que não possui ainda o implantado o PSF, mas realiza atendimento médico ambulatorial e possui o Programa dos Agentes Comunitários de Saúde (PACS).

Ao final de cada dia de trabalho de campo, os dados obtidos por cada pesquisador, por meio destes diferentes recursos, foram discutidos em minioficinas, configurando-se como uma pré-análise dos mesmos, bem como subsidiando o trabalho de campo do dia seguinte. Essa pré-análise permitiu a realização, ainda em campo, do primeiro desenho analisador do fluxo dos dados do SIAB e do SIS/HIPERDIA, facilitando a compreensão e o desenvolvimento deste estudo.

Foram feitas entrevistas abertas aos profissionais responsáveis pela alimentação do banco de dados com a finalidade de esclarecer o modo como o SIAB e o SIS/HIPERDIA são alimentados, bem como o modo como a informação neles contida é utilizada. Desta forma, participaram deste estudo o ACS e o enfermeiro da UBS, dois técnicos administrativos que atuam diretamente na alimentação do SIAB e SIS/HIPERDIA na Secretaria Municipal de Saúde de Alta Floresta-MT e o coordenador da Atenção Básica do município em questão.

As entrevistas com esses profissionais compuseram as Notas de Observação em Campo. As primeiras análises dos pesquisadores durante o período que estiveram reconstruindo a trajetória assistencial do usuário hipertenso compuseram as Notas de Diário de Campo. Todas as citações de Notas de Observação e Diário de Campo neste estudo foram provenientes do trabalho de campo, bem como, das discussões sobre os serviços de saúde realizados pelo grupo de pesquisadores.

Para melhor compreensão da produção, fluxo e análise dos dados do SIAB e SIS/HIPER- 
DIA, que não ficaram totalmente esclarecidas no município de Alta Floresta-MT, foi realizada uma visita a Secretaria de Estado de Saúde de Mato Grosso (SES/MT) para compreender o papel dessa Instituição que recebe as informações vindas dos municípios, fechando, assim, o fluxo de informações iniciado no município. Os registros dessa visita foram realizadas como Notas de Observação em Campo.

Os dados apreendidos a partir dessas estratégias metodológicas foram analisados tendo como base a realidade existente na produção de dados e seus usos, bem como aquilo que é preconizado pelo MS, ${ }^{8}$ resultando num desenho analítico do fluxo de dados do SIAB e do SIS/HIPERDIA, desde a produção das informações pelos ACSs até a chegada dos dados ao DATASUS. Este desenho ampliou as possibilidades de análise da configuração dos SIS no município, visto que permitiu uma leitura facilitada e uma melhor compreensão da seqüencia dos fluxos de dados dentro dos SISs.
Este estudo atendeu todos os aspectos éticos previstos na Resolução No 196/96 do Conselho Nacional de Saúde conforme a aprovação do projeto de pesquisa ao qual este estudo se vincula pelo Comitê de Ética em Pesquisa com Seres Humanos do Hospital Universitário Júlio Müller (HUJM), sob o Ofício N² 235/CEP-HUJM/2005.

\section{RESULTADOS E DISCUSSÃO}

Neste estudo evidenciamos que os dados que compõem o SIAB são gerados inicialmente nas UBSs, como informações detalhadas sobre as condições de vida e saúde das famílias. Desta forma, a Atenção Básica pode ser considerada como porta de entrada do usuário no SUS, bem como, da informação no SIS. Essas informações são produzidas pelo ACS, tanto de forma objetiva (numérica) quanto subjetiva. Contudo, a seguir essas informações passam por várias instâncias dentro do Sistema de Saúde, ganhando o formato de dado como uma expressão eminentemente numérica. ${ }^{1}$

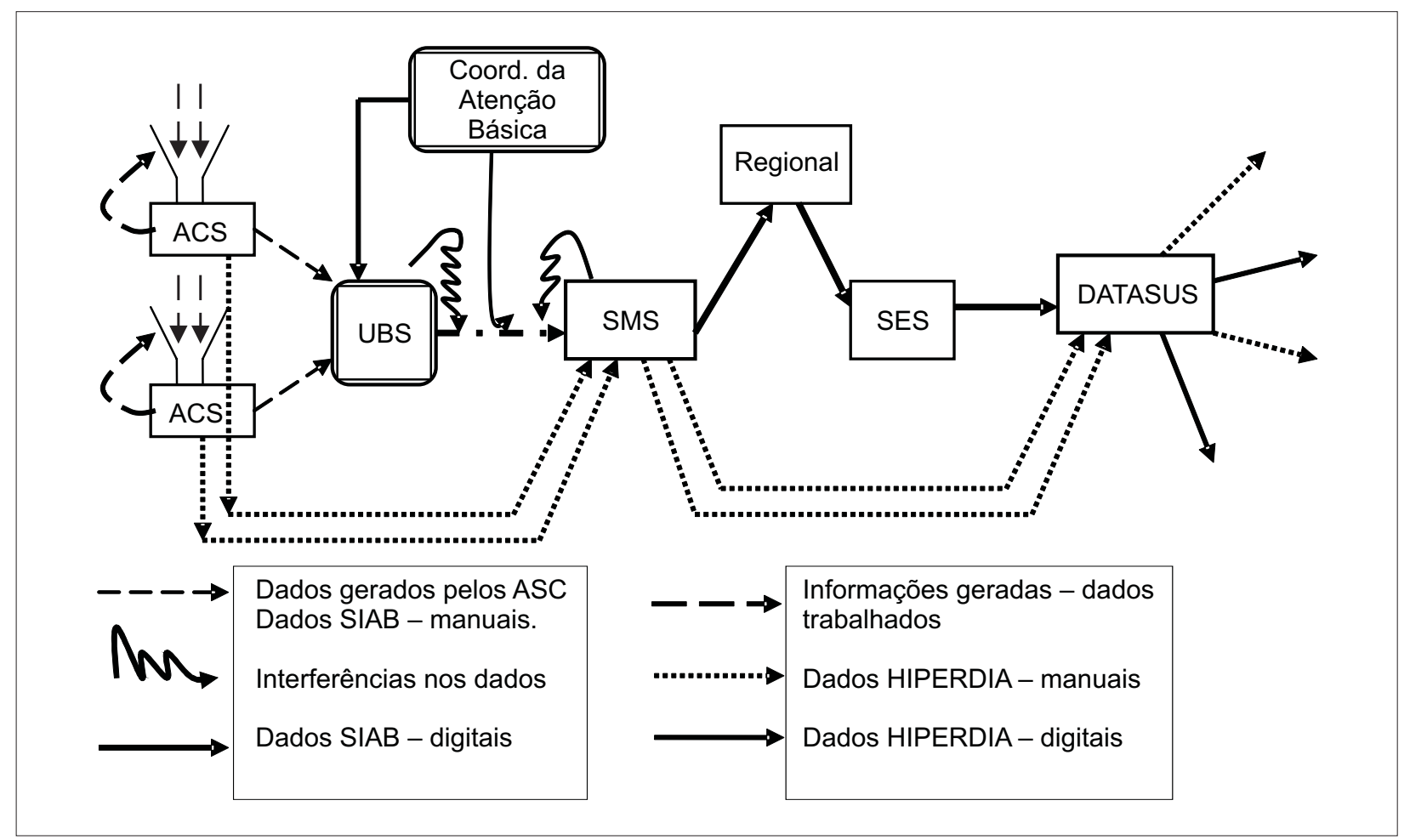

Figura 1 - Fluxo de dados do SIAB e do HIPERDIA do município de Alta Floresta ao DATASUS

A Figura 1, em sua primeira parte, indica que o fluxo dos dados tem início antes mesmo de chegar à UBS, quando diversas informações são produzidas pelos ACSs durante as visitas domiciliárias que realizam com as famílias dentro de suas micro-áreas. Desta forma, [...] os dados apreendidos são registrados em pequenos cadernos que possuem uma lógica própria do ACS, onde este anota nome e endereço do usuário e se o encontra em casa, coloca um OK na frente dos dados (Notas de Observação em Campo -18/03/2008).

Nesse caderno, são registradas muitas informações que não entram e/ou não se encaixam 
nos seus relatórios mensais, tal como o motivo de não ter realizado determinada visita domiciliar bem como informações que não são utilizadas pela UBS, mas reorientam o trabalho cotidiano do ACS. Tais informações poderiam ser valiosas no cuidado em saúde, pois, [...] muitas vezes as informações por ele geradas não são utilizadas devido à dificuldade de acesso ao enfermeiro e ao médico que o ACS tem nas unidades onde é implantado apenas o Programa de Agentes Comunitários de Saúde, mas não o PSF. O $A C S$ relatou ainda que, nos quatro anos em que trabalha no Centro de Saúde, teve contato direto com o médico da unidade apenas uma vez em uma palestra (Notas de Observação em Campo - 20/03/2008).

A distância do ACS em relação ao enfermeiro e ao médico do PACS faz com que muitas informações fiquem restritas aos cadernos de anotações dos próprios ACSs e que sejam utilizadas somente por eles. Da mesma forma, o estudo realizado aponta que os ACSs são os primeiros a produzirem informações, pois, transformam os dados apreendidos em suas visitas em algo com significado importante para a boa assistência em saúde dos usuários e para reconhecimento da população adstrita do município. ${ }^{1}$ Porém, $[$... $]$ o que o ACS repassa para a UBS é somente número e, embora sejam muitos os dados e as informações apreendidas pelo o ACS, a quantidade destes na composição dos relatórios das UBS é bem menor (Notas de Observação em Campo - 18/03/2008).

Nesse sentido, é como se as informações produzidas pelos ACS passassem previamente por filtros, antes mesmo de iniciarem seu fluxo no SIS, ou seja, as informações que podem alimentar o SIS são aquelas passíveis de quantificação, previamente definidas nestes sistemas, expressas em variáveis numéricas. Assim sendo, são privilegiados os relatórios que apresentam, essencialmente, a produção fixa e mensal da UBS, representando muito mais o trabalho e a produção do profissional, do que a condição de saúde dos usuários, ou mesmo a qualidade do atendimento ofertado pelo profissional e a resolutividade de sua prática em saúde.

A crítica aqui não é tanto em relação à utilização da expressão matemática (o número) para representar determinada realidade, mas a redução da diversidade de situações e condições de saúde à um único sistema de representação que possui, nele mesmo, suas próprias limitações no que se refere à diversidade e o significado qualitativo da realidade. Nesse sentido, os SISs necessitam ser repensados, pois, eles refletem a fragmentação da assistência sendo que deveria haver novas abor- dagens $^{3}$ e, que pudessem incluir as outras formas de informação produzidas pelo ACS e outros profissionais da área da saúde.

Nessa UBS, o acompanhamento dos usuários hipertensos é realizado pelos ACSs que realizam visitas domiciliárias, diferentemente do acompanhamento preconizado pelo Plano de Reorganização da Atenção a Hipertensão Arterial e Diabetes mellitus. ${ }^{7} \mathrm{O}$ ACS também é responsável por preencher as fichas de cadastramento do SIS/ HIPERDIA que [...] está suspenso na unidade (Notas de Observação em Campo - 18/03/2008). Mesmo sem este cadastramento e acompanhamento, considerados essenciais para aquisição e dispensa de medicação padronizada, ${ }^{7}$ os usuários hipertensos retiram a medicação no Centro de Saúde, pois, para isso, é necessário apenas apresentar o receituário médico e o cartão saúde na farmácia da UBS. No entanto, [...] quando o usuário estiver impossibilitado de ir à UBS para retirar a medicação, o ACS leva a medicação até sua residência, mas isso não é uma obrigatoriedade por não ser uma unidade do Programa de Saúde da Família (Notas de Observação em Campo - 18/03/2008).

As fichas de cadastramento das famílias do SIAB ficam com o ACS que, mensalmente, são entregues ao enfermeiro e, posteriormente, são enviadas para a SMS para serem digitadas, retornando para os ACSs logo após. Isso ocorre porque no município de Alta Floresta-MT não possui, ainda, informatização disponível na Atenção Básica. Este caso exemplar, evidencia as dificuldades encontradas na Atenção Básica que estão relacionadas à tecnologia da informação e a qualidade da informação em saúde. ${ }^{3}$

Na prática evidenciamos que: [...] as fichas cadastrais das famílias com os dados sobre sua situação de vida e saúde são preenchidas a lápis possibilitando a correção de algum erro quando houver necessidade. Os relatórios mensais dos ACS também são entregues para o enfermeiro para serem digitados. Diferentemente, as fichas do SIS/HIPERDIA são preenchidas em duas vias: uma fica na unidade de saúde onde foi gerada e a outra fica na SMS em grandes pastas de arquivo (Notas de Observação em Campo - 17/03/2008).

Antes dos dados chegarem a SMS para serem digitados e alimentar os SIS, ocorre uma primeira interferência nos dados pelo enfermeiro da UBS de modo a atender a formalidade dos SIS, conforme pode ser visualizado no fluxo analisado (Figura 1). Portanto, [...] os SIS não permitem todas as movimentações existentes dentro da área de abrangência de uma unidade, sendo que, por isso, o 
enfermeiro interfere nesse fluxo ocultando, por exemplo, a movimentação de uma gestante que chega ao município no sétimo mês de gestação para o nascimento de seu bebê e, após o parto, vai para outra unidade de saúde ou município. A interferência acontece, também, quando há o aumento e/ou diminuição dos membros de uma família em um curto período de tempo (Notas de Diário de Campo -18/03/2008).

Identificamos também, que a produção dos dados referente ao controle das medicações dispensadas pode ter falhas, pois, quando o usuário não apresenta o cartão de saúde, a dispensa da medicação não é registrada no seu prontuário. Isso se dá devido ao fato dos [...] prontuários serem identificados pelo número do cartão, deixando dúvidas de como é realizada a inserção destes dados nos relatórios (e1) e, inclusive para a alimentação do banco de dados.

Os dados que são enviados para a alimentação do SIAB e do SIS/HIPERDIA possuem uma fonte comum de produção de dados, o ACS. Porém, esses dados caminham em paralelo e não se cruzam durante o longo fluxo que percorrem. Os dados que alimentam o SIAB são referentes aos números de usuários hipertensos cadastrados na ficha de cadastramento das famílias. Enquanto que no SIS/HIPERDIA, os dados produzidos na UBS são referentes aos usuários hipertensos acompanhados mensalmente, sendo que esses registros demonstram o número de consulta e controle de dispensa das medicações padronizadas pelo Ministério da Saúde. ${ }^{6-7}$

Com base nisso, evidenciamos um dos primeiros problemas operacionais referente aos dados produzidos que alimentam tanto o SIAB como o SIS/HIPERDIA. Os dados por mais que tenham uma fonte comum, ambos não se cruzam para a realização de análise situacional em saúde e, muito menos, para a produção de informações sobre a resolutividade da atenção à saúde aos usuários hipertensos atendidos na UBS. O SIS/ HIPERDIA foi criado com o objetivo de poder planejar, monitorar e acompanhar os casos diagnosticados tanto de hipertensão arterial como de Diabetes mellitus e, inclusive, para o planejamento da assistência à saúde. Outro problema identificado foi que a ficha de cadastramento não tem sido utilizada, pois, a primeira via permanece no prontuário do usuário sendo que a mesma não é retroaliementada e, a segunda via é encaminhada para a SMS para ser arquivada após a alimentação do banco de dados, sendo que este posteriormente não é analisado. Outros problemas em relação à produção, fluxo e análise de dados são descritos em outros estudos, ${ }^{12-13}$ em relação às fichas de cadastro e de acompanhamento que são preenchidas adequadamente, sendo que tal fato poderá estar contribuindo para a dificuldade em construir um banco de dados para posterior análise situacional de saúde referente a essas doenças em questão.

Identificamos que se o usuário hipertenso e/ou diabético que não consultar e/ou não retirar a medicação por um longo período, como por exemplo, 60 dias, ele não aparecerá mais nos relatórios mensais do SIS/HIPERDIA, conforme o relato do técnico responsável por alimentar o SIS/HIPERDIA [...] porque se eu cadastrar aqui, se eu apenas cadastrar, o cadastro dele fica parado aqui, nem aparece nos relatórios (e2). Entretanto, no SIAB, [...] o mesmo usuário pode continuar a existir se ofuncionário que alimenta o sistema ou se o ACS não der baixa por sua ausência (Notas de Diário de Campo - 18/03/2009).

Outro motivo para dissensos entre dados desses SISs é que nem sempre o número de cadastrados corresponde ao número real de usuários hipertensos e diabéticos na área considerada. Isso pode ocorrer por falhas no cadastramento desses usuários ou, ainda, pela dificuldade na adesão do usuário ao acompanhamento na UBS. Em nossas observações constatamos que [...] esse acompanhamento se torna ainda mais difícil nas UBSs que ainda não têm implantado o Programa Saúde da Família, bem como por não haver uma prática de busca ativa dos faltosos e também devido a uma população flutuante considerável no município em questão (Notas de Diário de Campo - 18/03/2008).

Outro problema que surge com a divergência de dados entre os dois SISs é em relação à aquisição e dispensa de medicamentos para a Atenção Básica, sendo que este é, justamente, um dos principais objetivos do SIS/HIPERDIA. ${ }^{7}$ Como citado anteriormente, o usuário que não faz o acompanhamento regularmente na UBS poderá não aparecer nos relatórios do SIS/HIPERDIA, conforme relato do técnico que se [...] ela [usuária] fica cadastrada, mas ela fica é, é inativa dentro do Programa. [...] na hora de contar é, a quantidade de pacientes cadastrados para o envio de medicamentos, ela não vai aparecer e se ela não aparecer ela vai ficar sem medicamento (e2). E, se o usuário não consta nos relatórios poderá comprometer o planejamento da assistência, principalmente, em relação à dispensa de medicamento tanto para hipertensão arterial como para o Diabetes mellitus. O planejamento das ações assistenciais e da dispensa de medicamentos está relacionado ao número de usuários cadastrados na UBS.7-8 
Tanto na alimentação do SIAB quanto do SIS/HIPERDIA, [...] os técnicos detectam erros no preenchimento das fichas enviadas pelas UBS e lançam mão de duas formas para corrigir esses dados: eles podem entrar em contato com o enfermeiro da unidade, perguntar a respeito do dado que falta e preencher e/ou devolver a ficha para ser corrigida; ou o técnico se comunica diretamente como ACS através de bilhetes anexados às fichas a serem corrigidas. Tais procedimentos dão margem para alteração indiscriminada dos dados produzidos nas UBS (Notas de Diário de Campo - 21/05/2008).

Essas práticas de interferência nos dados podem ser interpretadas como uma forma de planificar as informações, ou seja, reduzir a um plano. ${ }^{14}$ Neste caso, o plano seria o modelo de SIS no qual todas as informações devem ser submetidas e modeladas ao que está pré-estabelecido. Os SISs, enquanto programas de informática e banco de dados, reconhecem apenas números (dados) na sua alimentação, tornando-se inflexíveis às informações de outra natureza, ainda que importantes para o planejamento da assistência à saúde. Assim, há uma grande valorização da produção de dados quantificáveis que possibilitam o planejamento e a análise de metas quanto ao fato de terem sido ou não atingidas pela UBS. Assim, os dados se restringem a quantificar consultas, exames e visitas domiciliárias realizadas, dentre outros itens de igual natureza.

Com base no que evidenciamos sobre o que é armazenado no computador do setor de SIS da SMS, são apenas dados numéricos que permanecem, visto que são representações da informação, e não a informação propriamente dita. ${ }^{1}$ Dessa forma, a configuração dos SISs não permite a apreensão da realidade de cada município e de cada UBS, de modo a expressar o que realmente ocorre nesses espaços e na vida da população. Como pudemos observar, [...] a realidade do município e da UBS é transformada em números e somente o município tem o poder de visualizá-la antes que se perca no conjunto dos números dos demais municípios da Regional de Saúde, do Estado e do País (Notas de Diário de Campo - 21/05/2008).

A formalização exigida pelo SIS tem gerado uma pressão que influencia na prática dos profissionais de saúde, sendo que essa pressão é fruto de uma cobrança quanto a produção exigida, metas a serem atingidas e a quantidade de dados a serem gerados pela UBS. Os profissionais de saúde trabalham para gerar a informação que o SUS necessita e isso leva os diferentes níveis de atenção à saúde a planificar a informação, conforme o relato do profissional: [...] eu já fico preparado [...] eu já tenho que ter a justificativa (e1). A fala desse profissional que alimenta o SIAB demonstra que, muitas vezes, os técnicos enviam seus dados esperando uma repreensão por parte da próxima instância a recebê-los. No município de Alta Floresta-MT, os dados do SIAB ainda são enviados para a SES que, após conferência, os enviam para o MS para compor a base do DATASUS.

Os dados que alimentam o SIS/HIPERDIA são enviados diretamente dos municípios para o MS e deste para o DATASUS. Evidenciamos que [...] a SES, por sua vez, faz apenas um acompanhamento dos dados enviados ao Ministério da Saúde, bem como realiza cobranças das unidades de saúde em casos de erros ou atrasos na produção e envio dos relatórios (Notas de Diário de Campo - 21/05/2008). O que retorna para os municípios são apenas as cobranças a respeito de dados que não são enviados e os prazos a serem cumpridos para que não haja corte no repasse de recursos ao município. Os dados que saem das UBS tornam-se invisíveis à medida que avançam na escala da hierarquia do fluxo do SIS, sendo que [...] a medida que os dados sobem na hierarquia, as portas se fecham mais (Notas de Diário de Campo - 21/05/2008).

O modo como se configura o fluxo da informação aqui analisado diverge da proposta do MS que visa disponibilizar os dados doSIAB para fornecer informações que embasem a tomada de decisão pelos gestores do SUS, bem como, possibilitar a instrumentalização das instâncias de Controle Social disponibilizando, assim, os dados para o uso de todos os envolvidos na consolidação do SUS.,15 Tal proposta não é plenamente alcançada, uma vez que os dados do SIAB não são totalmente visibilizados no nível da SES, podendo esta instância ver apenas os dados já condensados nos relatórios mensais.

É importante lembrar que a finalidade da informação em saúde é identificar problemas individuais e coletivos da população, servindo de base para análise da situação encontrada e subsidiando a busca de alternativas para solucionar tais problemas. ${ }^{16}$ Se as informações não chegam aos demais níveis de atenção à saúde, cabe questionar: como a SES desenvolve estratégias para o fortalecimento da gestão de políticas de prevenção e tratamento da hipertensão arterial e Diabetes mellitus no estado, prevista na Portaria $N^{0} 371$ do $\mathrm{MS}^{15}$ se cabe a SES apenas o papel de acompanhar o envio de relatórios, sem que tenha acesso aos dados do município, como o Estado tem pleno conhecimento 
da situação de saúde de seus municípios se não visualiza e analisa completamente os dados que mostram a realidade da saúde dos mesmos?

Ainda que os dados fossem visualizados pela SES, sua atribuição prevista pelo $\mathrm{MS}^{15}$ se torna fragilizada pelo fato da produção, fluxo e análise da informação em saúde chegar até ela somente sob forma de números, com possibilidade restrita de expressar qualitativamente a situação de saúde.

No fluxo que os dados percorrem, apenas [...] o município visualiza a informação de modo mais consistente através da Coordenação da Atenção Básica, visto que ainda trabalha elementos mais amplos que apenas os numéricos, o que lhe permite visualizar como está à saúde em seu município (Notas de Diário de Campo - 18/03/2008). E a informação produzida a partir das UBS, ao passar do nível da Coordenação da Atenção Básica, invisibiliza os usuários que deixam de ser uma realidade viva para se tornarem números apenas, que passarão a representar a produção das práticas profissionais, ou seja, números de consultas, números de medicamentos dispensados, números de cirurgias, etc.

O SIS é pouco sensível a expressão qualitativa da realidade das unidades de saúde e dos municípios, fazendo com que os dados sirvam apenas para mostrar a produção quantitativa dos mesmos, gerando uma pressão para que estes estejam sempre dentro do plano e metas traçados pelas instâncias hierarquicamente acima de quem lida diretamente com a realidade de vida e saúde da população.

Outra constatação importante é que o usuário é invisibilizado no fluxo dos dados, que deixam de expressar suas diversas necessidades, transformando-se em um número. Número este que, muitas vezes, nem é real, já que o mesmo usuário pode entrar várias vezes no sistema de informação através do diversos SISs existentes no país. Uma única mulher, por exemplo, pode entrar noSIS/Pré Natal, no SIS/Colo, no SIS/HIPERDIA e no SIAB. Porém, em cada SIS ela é um número, apesar de ser uma única pessoa, sendo que esse fato decorre do não partilhamento dos dados dos SISs. Portanto, o SIS reflete a fragmentação da assistência e o quanto ele ainda não permite uma composição de dados que refletiam a situação de saúde.

\section{ALGUMAS CONSIDERAÇÕES FINAIS}

O presente estudo revelou que, apesar de sua importância para a saúde, os SISs ainda apresentam pontos de estrangulamentos na sua forma de organização e prática, o que compromete a confiabilidade dos dados aí produzidos, visto não representarem a realidade da situação de saúde da população.

Os SISs foram criados com o intuito de acompanhar a produção de dados para assegurar avaliações da situação de saúde de toda a população e, assim, servirem como base para o planejamento do nível local como um instrumento para as práticas de atenção e de gestão. Porém, isso não ocorre na realidade, conforme pudemos constatar em nosso trabalho de campo. O que pudemos perceber é que os dados produzidos nas UBSs são pouco trabalhados e pouco utilizados como ferramentas de melhoria e planejamento da assistência à saúde da população. Assim como na assistência, os SISs deveriam ter seu foco na população e suas necessidades, retornando todos esses dados sob forma de políticas que garantam uma atenção à saúde de qualidade e resolutiva aos usuários, que são a razão de ser de todo serviço de saúde.

Este estudo apresentou sua limitação referente à literatura que discuta e reflita sobre a produção, fluxo e análise dos dados do SIS visto ser ainda escassa. Especialmente em relação ao SIAB e SIS/HIPERDIA, embora possuam uma mesma origem quanto a fonte e produção de dados, seguem de forma isolada, uma vez que esses SISs não são compartilhados. Essa situação, inclusive com divergência de informações, torna dificultado o planejamento da assistência aos usuários com hipertensão arterial e Diabetes mellitus.

Mas este estudo possibilitou dar visibilidade ao modo como as práticas profissionais se homogeneízam no SIS e, como o usuário é invisibilizado em relação as suas necessidades de saúde, pois as mesmas não são consideradas como dados naquilo que é pré-definido como alimentador desse sistema, havendo ainda, dados que se perdem ao longo do fluxo de alimentação destes. Assim, este estudo nos convida a refletir sobre a importância da informação qualitativa que possa expressar as necessidades ampliadas de saúde para se pensar em integralidade e resolutividade na atenção à saúde.

\section{REFERÊNCIAS}

1. Setzer VW. Dado, informação, conhecimento e competência. Data Grama Zero. Ciência 1999 Dez [acesso 2008 Jun 30]; Disponível em: http:/ / www. datagramazero.org.br/dez99/index.htm

2. Moraes IHS. Informações em saúde - da prática fragmentada ao exercício da cidadania. São Paulo (SP): Hucitec/Abrasco; 1994. 
3. Moraes IHS, Santos SRFR. Informações para gestão do SUS: necessidades e perspectivas. Informe epidemiológico do SUS. 2001 Jan-Mar; 10(1):49-56.

4. Silva AS, Laprega MR. Avaliação crítica do sistema de informação da atenção básica (SIAB) e de sua implantação na região de Ribeirão Preto, São Paulo, Brasil. Cad Saúde Pública. 2005 Nov-Dez; 21(6):1821-8.

5. Ministério da Saúde (BR). Sistema de Informação da Atenção Básica [página da internet]. Brasília (DF): MS. [acesso 2008 Abr 14]. Disponível em: http:// www.datasus.gov.br/siab/siab.htm

6. Ministério da Saúde (BR). Secretaria de Atenção à Saúde. Departamento de Atenção Básica. SIAB manual do sistema de informação da atenção básica. Brasília (DF): MS; 2003.

7. Ministério da Saúde (BR). Secretaria de Políticas Públicas. Plano de Reorganização da Atenção à Hipertensão Arterial e Diabetes Mellitus. Rev Saúde Púb. 2001 Dez; 35(6):585-8.

8. Ministério da Saúde (BR). Portaria Conjunta $N^{\circ} 112$, de 19 de junho de 2002. Dispõe sobre o fluxo de alimentação da base nacional do Hiperdia. Brasília (DF): MS; 2002 [acesso 2008 Jun 3]. Disponível em: http://hiperdia.datasus.gov.br/manuais/portaria conjunta 112.doc

9. Minayo MCS. O desafio do conhecimento: pesquisa qualitativa em saúde. $9^{a}$ ed. São Paulo (SP): Hucitec; 2006.
10. Faria APS. A Experiência de adoecimento e a busca por cuidado de uma pessoa com diabetes mellitus [dissertação]. Cuiabá (MT): Universidade Federal de Mato Grosso. Programa de Pós-Graduação em Enfermagem; 2007.

11. Thaines GHLS, Bellato R, Faria APS, Araújo LFS. A busca por cuidados empreendida por um usuário com Diabetes mellitus: um convite à reflexão sobre integralidade em saúde. Texto Contexto Enferm. 2009 Jan-Mar; 18(1):57-66.

12. Ferreira CLRA, Ferreira MG. Características epidemiológicas de pacientes diabéticos da rede pública de saúde - análise a partir do HIPERDIA. Arq Bras Endocrinol Metab. 2009 Jan-Fev; 53(1):80-6.

13. Oliveira CA, Palha PF. Sistema de informações HIPERDIA, 2002-2002, adequação das informações. Cogitare Enferm. 2008 Jul-Set; 13(3):395-402.

14. Ferreira ABH. Minidicionário Aurélio. $6^{\mathrm{a}}$ ed. Curitiba (PR): Posigraf; 2004.

15. Ministério da Saúde (BR). Portaria No 371/GM de 4 de março de 2002. Dispõe sobre o Programa Nacional de Assistência Farmacêutica para Hipertensão Arterial e Diabetes mellitus. Brasília: Diário Oficial da União de 6 de março de 2002, Seção 1, página 88. [acesso 2008 Jun 3]. Disponível em: http:/ / hiperdia.datasus.gov. $\mathrm{br} /$ manuais/portaria ministerial371.zip

16. Branco MAF. Sistemas de informação em saúde no nível local. Cad. de Saúde Púb. 1996 Abr-Jun; 12(2):267-70. 